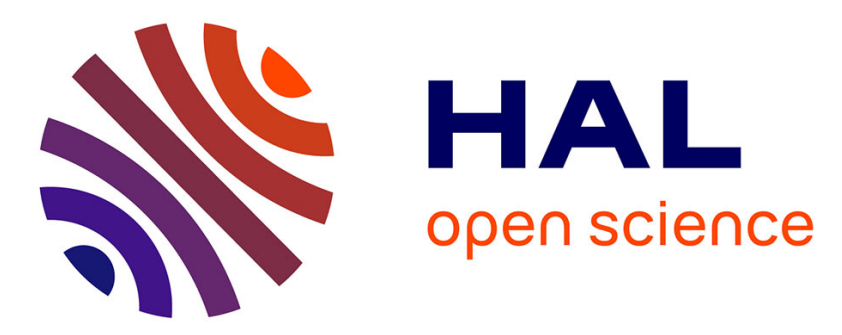

\title{
ANALYSE EBIC DES JOINTS DE GRAINS DANS LE SILICIUM POLYCRISTALLIN
}

\author{
P. Ruterana, A. Bary, G. Nouet
}

\section{To cite this version:}

P. Ruterana, A. Bary, G. Nouet. ANALYSE EBIC DES JOINTS DE GRAINS DANS LE SILICIUM POLYCRISTALLIN. Journal de Physique Colloques, 1982, 43 (C1), pp.C1-27-C1-31. 10.1051/jphyscol:1982105 . jpa-00221758

\section{HAL Id: jpa-00221758 https://hal.science/jpa-00221758}

Submitted on 1 Jan 1982

HAL is a multi-disciplinary open access archive for the deposit and dissemination of scientific research documents, whether they are published or not. The documents may come from teaching and research institutions in France or abroad, or from public or private research centers.
L'archive ouverte pluridisciplinaire HAL, est destinée au dépôt et à la diffusion de documents scientifiques de niveau recherche, publiés ou non, émanant des établissements d'enseignement et de recherche français ou étrangers, des laboratoires publics ou privés. 


\title{
ANALYSE EBIC DES JOINTS DE GRAINS DANS LE SILICIUM POLYCRISTALLIN
}

\author{
P. Ruterana* , A. Bary ${ }^{*}$ et G. Noue $t^{* *}$ \\ * Equipe Physique du Solide \\ * Equipe Matériaux-Microstructure
}

Laboratoire de Cristallographie, Chimie et Physique du Solide, I,A 251, ISMRA, Université de Caen, 14032 Caen Cedex, France

Résumé. - Du silicium polycristallin est examiné au TEM pour la caractérisation cristallographique des joints et au SEM en mode EBIC pour la détermination de leur activité électrique. Les plans de macle cohérents sont trouvés être électriquement inactifs à la différence des autres joints qui sont constitués par de petites facettes ou décorés par des précipités.

Abstract. - Grain boundaries in polycristalline silicium have been studied using EBIC, SEM and TEM. The reduction of the electrical properties is due to the presence of defects in the grain boundaries structure. These defects, dislocations or facets, are evidently associated with segregated atoms.

1. Introduction. - La dimension des cellules solaires a eu un impact immédiat sur le coût de leur production. De ce fait le silicium monocristallin de très grande qualité électronique a été remplacé par du matériau polycristallin. Actuellement, il apparaît que la structure optimale soit réalisée par des grains colonnaires. Ce remplacement s'est accompagné d'une baisse de rendement des photopiles liées à la présence de ces joints. La modification du courant induit (EBIC) à la traversée de ces joints est attribuée aux défauts et impuretés situés dans ces joints $(1,2,3)$. L'objectif de cette étude est de relier la cristallographie du joint à son comportement électrique.

\section{Matériau et Techniques opératoires (SEM, EBIC et TEM)}

Le matériau utilisé est du type silso fourni par le Société Wacker. La cellule solaire est constituée par une jonction $p-n$ située à $0,4 \mu n$ de profondeur, $1^{*}$ épaisseur totale étant de $300 \mu m$. Les mesures relatives au comportement électrique des joints de grains sont effectuées au microscope électronique à balayage (SEM) selon deux modes : electrons retrodiffusés pour visualiser les grains et leurs joints, courant induit (EBIC) pour mesurer l'intensité du courant de diffusion collecté aux bornes de la cellule. Sur une même plaque photographique les deux phénomènes sont superposés, une ligne horizontale permet de répérer le balayage. Après ces mesures en mode induit une rondelle de $3 \mathrm{~mm}$ de diamètre est découpée, amincie mécaniquement à une centaine de microns et finalement amincie chimiquement ou ioniquement. La face "avant" de la cellule, celle qui est la plus proche de la jonction, reste intacte, toutes les opérations d'amincissement sont effectuées par la face arrière.

Au microscope électronique à transmission (TEM) les grains contigus sont orientês au moyen d'une méthode décrite par ailleurs et les indices des plans de l'interface sont déterminês (4).

Un même joint est donc caractérisé électriquement (EBIC + SEM) et cristallographiquement (TEM). Il faut remarquer cependant, que les grossissements sont fortement différents : EBIC + SEM, X SO, TEM x 10000 à 30000 et que les résolutions de ces deux techniques ne scnt pas comparables.

3. Caractéristiques électriques d'une cellule solaire polycristalline

En comparaison avec une celluie solaire monocristalline les caractêristiques du matêriau polycristallin sont inférieures (Tableau l). 


\begin{tabular}{|l|c|c|c|c|c|}
\hline & $\mathrm{L}_{\mathrm{D}}^{\mu \mathrm{m}}$ & $\mathrm{ICC}_{\mathrm{mA} / \mathrm{cm}^{2}}$ & $\mathrm{~V}_{\mathrm{co}} \mathrm{mV}$ & $\mathrm{F} \cdot \mathrm{R}$ & $\mathrm{M} \%$ \\
\hline Si mono & $>100$ & 28 & 600 & 0.75 & 12 \\
\hline Si poly & $<50$ & 23 & 530 & 0.69 & 8 \\
\hline
\end{tabular}

Table I : mono and poly - Si characteristics

Tableau I : comparaison des caractéristiques

$L_{D}$ : longueur de diffusion, ICC : courant de court-circuit, $V_{C O}$ : tension de circuit ouvert, F.R, : facteur de remplissage, $M$ : rendement.

Une analyse de $l^{\prime}$ influence des joints de grains sur le comportement électrique des cellules a été entreprise.

\section{Structure cristallographique du matériau.}

La figure l obtenue au SEM en mode électrons rêtrodiffusés, correspond à un carré de $2 \mathrm{~cm}$ de côté d'une cellule. Cette première observation indique la présence de deux groupes d'interfaces : les uns parfaitement rectilignes, parallèles entre eux (groupe 1 ) et les autres aux contours plus ou moins courbes (groupe 2). On peut, dès à présent, remarquer que le groupe 1 est toujours situé à l'intérieur des grains définis par les joints du groupe 2. Le diamètre moyen de ces grains est voisin de $1,2 \mathrm{~mm}$.

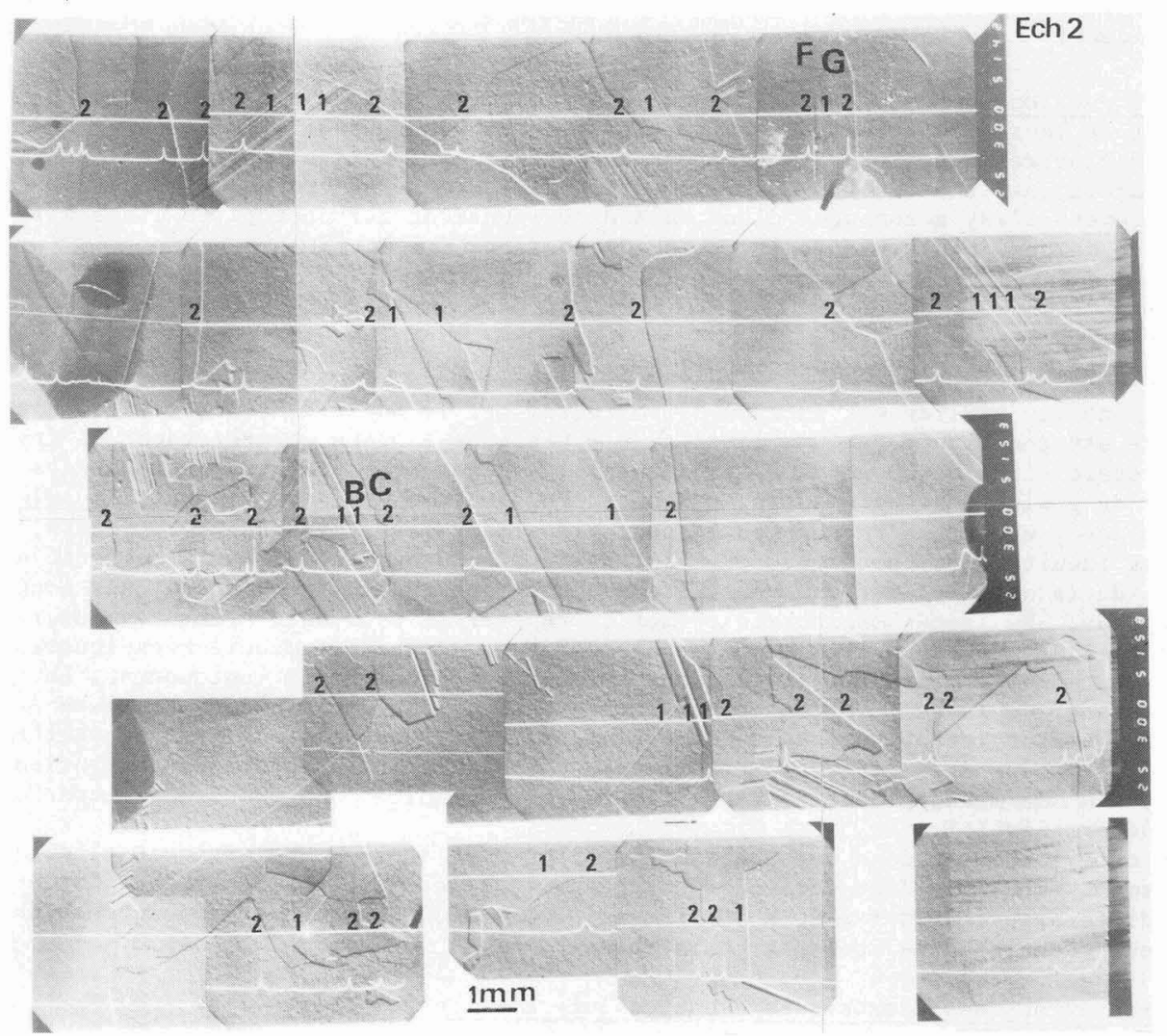

Fig. 1 : enregistrement du signaI EBIC

Fig. 1 : EBIC signal in poly - Si 
Au TEM deux échantillons prélévés dans la plaque voisine et ayant la même morphologie dûe à la structure colonnaire des joints ont été analysés systématiquement : détermination de la relation d'orientation entre grains contigus, de l'indice de coïncidence, des indices cristallographiques du plan du joint et du plan moyen de la lame (inclinaison $0^{\circ}$ ) (Tableau 2).

\begin{tabular}{|c|c|c|c|c|c|c|c|c|c|c|}
\hline E & $A / B$ & $\mathrm{~B} / \mathrm{C}$ & $\mathrm{C} / \mathrm{D}$ & $\mathrm{D} / \mathrm{E}$ & $\mathrm{E} / \mathrm{F}$ & $F / G$ & $\mathrm{G} / \mathrm{H}$ & $\mathrm{H} / \mathrm{I}$ & $\mathrm{H} / \mathrm{J}$ & $\mathrm{H} / \mathrm{K}$ \\
\hline $\begin{array}{l}a \\
b \\
c\end{array}$ & $\left|\begin{array}{c}3 \\
111 \\
211 / 111\end{array}\right|$ & $\begin{array}{c}3 \\
111 \\
111 / 001\end{array}$ & $\begin{array}{c}47 a \\
f a c \\
100 / 001\end{array}$ & $\begin{array}{c}3 \\
111 \\
001 / 111\end{array}$ & $\begin{array}{c}27 b \\
\mathrm{fac} \\
111 / 110\end{array}$ & $\begin{array}{c}27 b \\
? \\
110 / 111\end{array}$ & $\begin{array}{c}3 \\
111 \\
111 / 001\end{array}$ & $\begin{array}{c}3 \\
111 \\
001 / 110\end{array}$ & $\begin{array}{c}3 \\
111+211 \\
001 / 110\end{array}$ & $\begin{array}{c}19 b \\
? \\
001 / 221\end{array}$ \\
\hline & $\mathrm{A} / \mathrm{B}$ & $\mathrm{B} / \mathrm{C}$ & $\mathrm{C} / \mathrm{D}$ & $\mathrm{B} / \mathrm{D}$ & $\mathrm{F} / \mathrm{G}$ & $\mathrm{F} / \mathrm{H}$ & $\mathrm{G} / \mathrm{H}$ & $\mathrm{H} / \mathrm{I}$ & $\mathrm{I} / \mathrm{J}$ & $\mathrm{J} / \mathrm{K}$ \\
\hline $2 \mid \begin{array}{l}a \\
b \\
c\end{array}$ & $\begin{array}{c}3 \\
111 \\
001 / 111\end{array}$ & $\begin{array}{c}9 \\
111 / / 511 \\
111 / 001\end{array}$ & $\begin{array}{c}3 \\
111+211 \\
001 / 001\end{array}$ & $\begin{array}{l}27 \\
111+\mathrm{fac} \\
111 / 001\end{array}$ & $\begin{array}{c}27 b \\
f a c \\
001 / 111\end{array}$ & $\begin{array}{c}81 \\
? \\
001 / 001\end{array}$ & $\begin{array}{c}3 \\
111 \\
111 / 001\end{array}$ & $\begin{array}{c}3 \\
111 \\
001 / 001\end{array}$ & $\begin{array}{c}3 \\
111 \\
\infty\end{array}$ & $\begin{array}{c}3 \\
111 \\
111 / 001\end{array}$ \\
\hline
\end{tabular}

Table 2 : Cristallographic characterization

Tableau 2 : Caractérisation cristallographique

$a$ : indice de coincidence, $b$ : indices cristallographiques du plan du joint (fac : petites facettes, ? : non déterminés), $c$ : indices cristallographicues du plan moyen $\mathrm{J}$ : joint, $\mathrm{E}$ : Echantillon.

Ces résultats limitês confirment les observations déjà dêcrites (5). La majorité des interfaces $d^{\prime}$ indice de coindicence 3 , à l'exception des interfaces $(\mathrm{H} / \mathrm{J})_{1}$ et (C/D) 2, est constituée par des plans de macle (1II) et correspond au groupe 1. Les interfaces dont les indices de coïncidence sont des multiples de 3 sont généralement dûs aux différentes réactions possibles entre les macles du premier ordre et des ordres suivants (5). Par contre les autres interfaces ( $(C / D)_{1}$ et $\left.(H / K) !\right)$ d'indices de coincidence "quelconques" et les interfaces $(H / J)_{1}$, et $(C / D)_{2}$, macle du ler ordre, et $(B / C) 2$, macle du 2ème ordre sont a classer dans le groupe 2 caractérisé par des joints plus ou moins courbes à l'échelle du SEM. A l'échelle du TEM ces joints sont soit constitués par de grandes facettes (111) et (211) (macle du lex ordre), soit par de petites facettes (111) et d'autres à indices cristallographiques non déterminés (macle du 2ème oràre) soit par des facettes très petites d'indices "quelconques" pour les indices de coincidence 19 et 47.

\section{Analyse EBIC}

I'enregistrement de l'analyse EBIC sur la figure 1 confirme l'existence de ces deux groupes : le groupe 1 constitué par les plans de macle (111) ne modifie pas le courant EBIC à la différence du groupe 2. Une analyse plus systématique des différents joints d'un même grain montre un comportement différent selon les facettes examinées (Figure 2). Un traitement semi-quantitatif de ces informations est en cours de développement.

Trois échantillons dont deux sont notés $(B / C)_{2}$ et $(F / G)_{2}$ sur la fiqure 1 ont été examinés au TEM après analyse EBIC. Ces deux joints sont constitués de petites facettes et des dislocations extrinsèques sont présentes (Fig. 3). Leurs indices de coincidence sont respectivement 9 et $27 \mathrm{~b}$.

Dans le troisième échantillon le joint est décorê par des précipités ayant un diamètre moyen de $50 \mathrm{~nm}, 1$ 'indice de coincidence est alors de $19 \mathrm{~b}$.

\section{Discussion et Conclusion}

Les interfaces qui se forment pendant le refroidissement peuvent être classés en deux groupes. Ils apparaissent probablement de manière simultanée mais cependant leurs origines sant totalement différentes. Les plans de macle (111) (groupe 1) ne se forment qu'à l'intérieur des grains dont les joints constituent le groupe 2. Ces plans de macle sont associés aux macles de croissance dont la formation s'explique par un mécanisme de dislocation. Inversement, la formation des joints du groupe 2 

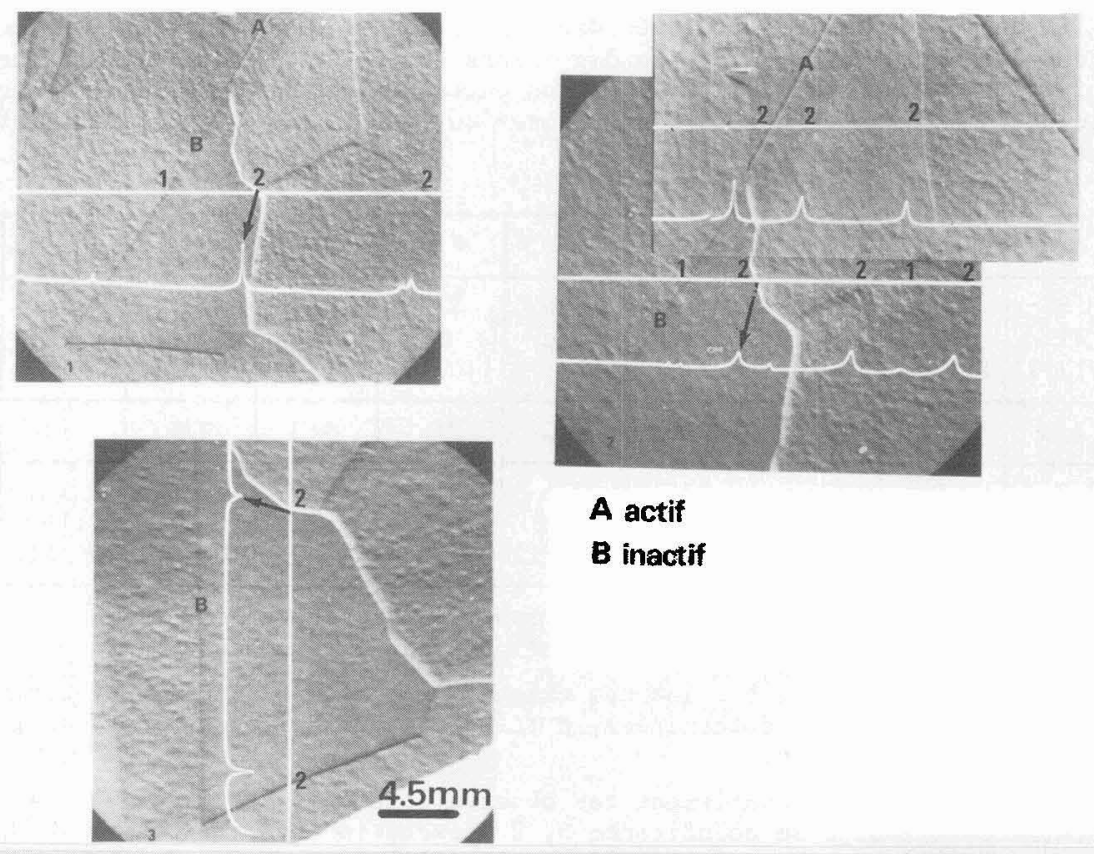
A actif
$B$ inactif
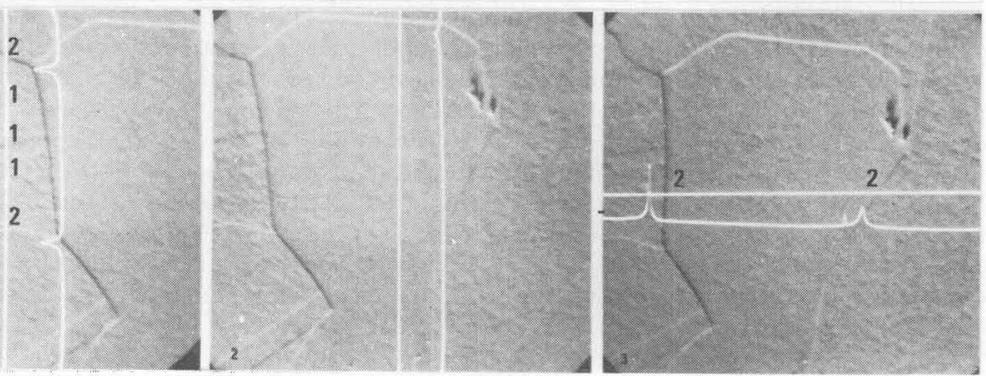

Fig. 2 : Variation de la diminution du courant induit selon las facettes.

Fig. 2 : Variation of the induced current reduction with respect to the orientation facets.

est associée directement à la solidification du lingot. Ces joints sont alors des lieux privilégiés pour la ségrégation des impuretés. Il est donc clair que les défauts de structure du joint de grains associés aux atomes segrégés favoriseront la recombinaison des porteurs minoritaires.

\section{7 . Remerciements}

Nous tenons à remercier la Radiotechnique Compelec Caen pour sa collaboration. 


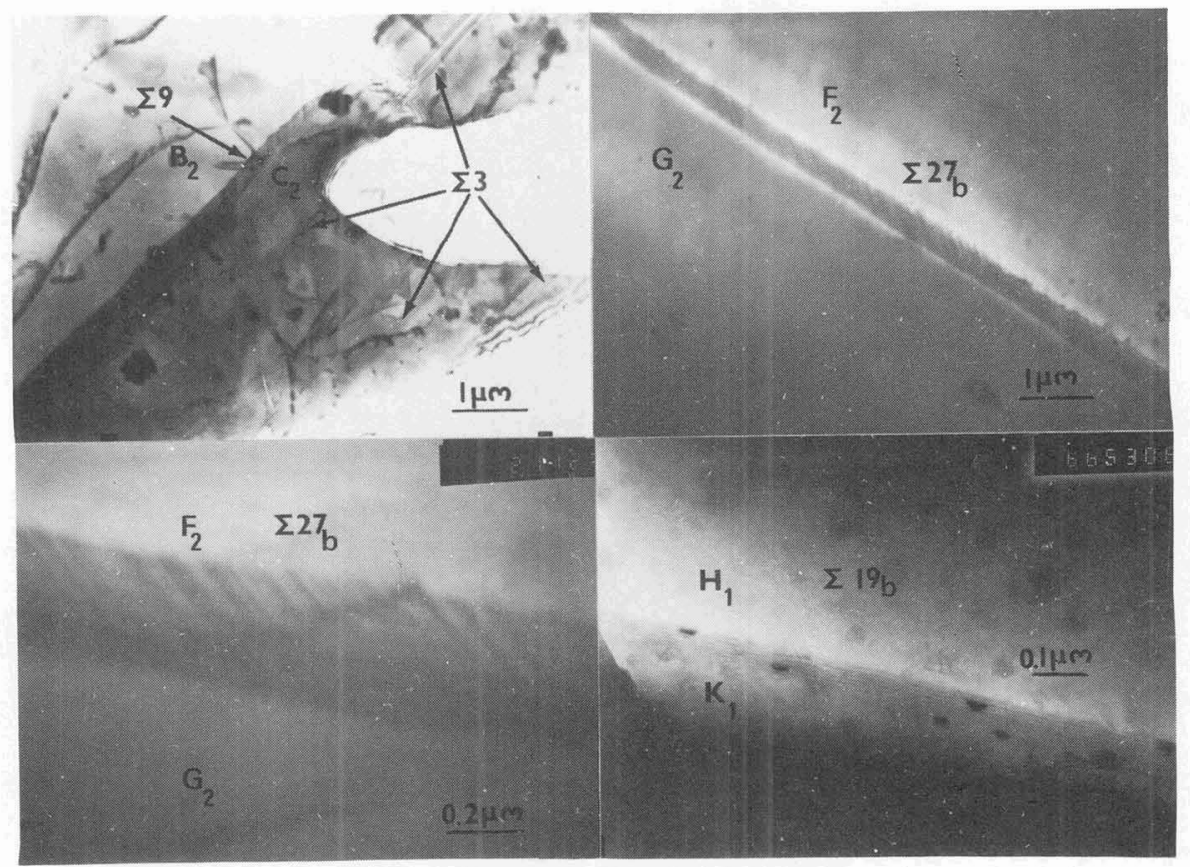

Fig. 3 : Défauts et précipités dans des joints ayant entraînê une diminution du courant EBIC.

Fig. 3 : Defects and precipitates in electrically active grain boundaries.

\section{Rêfërences}

1. STRUNK H., CUNNINGHAM B. et AST D., M.R.S. Conference (1980) Boston.

2. RALLON O., AUCOUTURIER M., HERVO C., MAUTREF M. et BELOUET C., J.M.A., Mém. Et. Scient., Revue de Métallurgie, 9 (1981) 410.

3. REDFIELD D., App 1. Phys. Lett. 40 (1982) 163.

4. KARAKOSTAS Th., NOUET G., BLERIS G.L., HAGEGE S. et DELAVIGNETTE P., Phys. Stat. Sol. (a), 50 (1978) 703 .

5. FONTAINE C. et ROCHER A., J. Microscopy 118 (1980) 105. 